\section{Varenicline: quantifying the risk}

The finding by Singh and colleagues that varenicline is associated with an increased risk of serious adverse cardiovascular events ${ }^{1}$ is not surprising because varenicline is frequently associated with hypertension. ${ }^{2,3}$ An elevation in blood pressure, however small, increases the risk of adverse cardiovascular outcomes. ${ }^{4}$ Before approving varenicline, the US Food and Drug Administration (FDA) should have mandated that the manufacturer provide the FDA with data on cardiovascular safety.

\section{Robert P. Blankfield MD MS}

Case Western Reserve University School of Medicine, Cleveland, Ohio

\section{References}

1. Singh S, Loke YK, Spangler JG, et al. Risk of serious adverse cardiovascular events associated with varenicline: a systematic review and meta-analysis. CMAJ 2011;183:1359-66.

2. Physician's desk reference. 64th ed. Montvale (NJ): Thomson; 2010. p. 2715.

3. Drug facts and comparisons. St. Louis (MO) Facts and Comparisons; 2011. p. 1115.

4. Weber MA. The ALLHAT report: a case of information and misinformation. J Clin Hypertens (Greenwich) 2003;5:9-13.

\section{CMAJ 2011. DOI:10.1503/cmaj.111-2062}

Singh and colleagues found that varenicline was associated with a significantly increased risk of serious adverse cardiovascular events compared with placebo (1.06\% [52/4908] in the group receiving varenicline v. $0.82 \%$ [27/3308] in that receiving placebo (odds ratio [OR] 1.72, 95\% confidence interval [CI] 1.092.71). ${ }^{1}$ They used the Peto method to calculate ORs and $95 \% \mathrm{CI}$, stating that the Peto method provides the best CI coverage and is more powerful and relatively less biased than the randomeffects analysis when dealing with low event rates.

The approximation used to calculate the $\log$ OR works well when the effects of intervention are small (i.e., ORs are close to 1.0), events are not particularly common and the studies have similar numbers in the experimental and control groups. ${ }^{2}$ As these criteria are not always fulfilled, the Peto method is not recommended as a default approach for analysis because it has been shown to give biased answers. On the other hand, when data are sparse (event rates are low or study size is small), MantelHaenszel methods have been shown to have better statistical properties. ${ }^{2}$

Sensitivity analyses by Singh and colleagues using the reciprocal of the treatment arm with a continuity correction (fixed Mantel-Haenszel OR 1.67, 95\% CI 1.06-2.64) or without a continuity correction (fixed MantelHaenszel OR 1.77, 95\% CI 1.09-2.88) showed results similar to those in the preliminary analysis using the Peto method. However, our recalculation of the same dataset as those in Figure 2 in Singh and colleagues' article using RevMan showed a statistically insignificant increase in serious adverse cardiovascular events with varenicline compared with placebo (fixed MantelHaenszel OR 1.56, 95\% CI 0.99-2.44).

This should be a proper result of the primary analysis in the meta-analysis. Therefore, there are no safety concerns about the potential for an increased risk of serious adverse cardiovascular events associated with the use of varenicline among tobacco users.

\section{Hisato Takagi MD PhD}

Takuya Umemoto MD PhD

Department of Cardiovascular Surgery, Shizuoka Medical Center, Shizuoka, Japan

\section{References}

1. Singh S, Loke YK, Spangler JG, et al. Risk of serious adverse cardiovascular events associated with varenicline: a systematic review and meta-analysis. CMAJ 2011;183:1359-66.

2. Deeks JJ, Higgins JP, Altman DG, editors. Chapter 9: Analysing data and undertaking meta-analyses. In: Higgins JP, Green S, editors. Cochrane handbook for systematic reviews of interventions version 5.1.0 (updated March 2011). Available: www .cochrane-handbook.org (accessed 2011 July 5).

CMAJ 2011. DOI:10.1503/cmaj.111-2063

We agree that there is a lack of clarity in Singh and colleagues' article; it is very difficult for readers to follow how the various assumptions and extrapolations have been made. ${ }^{1}$

The rather alarming figure of a $72 \%$ increase in serious cardiovascular events has been picked up by the media. However, in absolute terms, the increase in risk is only $0.24 \%$, which computes to an NNH (number needed to harm) of about 400. This is a very small increase in risk compared with the benefits of quitting smoking (number needed to treat 10 for varenicline).

Admittedly, Singh and colleagues' study may signal the need for caution when using varenicline in patients with a history of cardiovascular or active disease. The authors need to offer more explanation about how they reached the estimate of $\mathrm{NNH}$ of 28 , which appears to be an extrapolation of their findings to a population at very high risk of cardiovascular disease.

David J. Woods BSc MPharm

Consultant pharmacist

Mark D. Caswell BSc

Medical writer

Best Practice Advocacy Centre, Dunedin, NZ

\section{Reference}

1. Singh S, Loke YK, Spangler JG, et al. Risk of serious adverse cardiovascular events associated with varenicline: a systematic review and meta-analysis. CMAJ 2011;183:1359-66.

\section{CMAJ 2011. DOI:10.1503/cmaj.111-2064}

In their sophisticated review and metaanalysis, Singh and colleagues calculated the absolute risk for varenicline as $1.06 \%$ and that for placebo at $0.82 \%{ }^{1}$ Hence, the correct absolute risk elevation (ARE) is the difference between the two percentages $(0.24 \%$, or 0.0024$)$, which they also calculated correctly.

However, in their interpretation, Singh and colleagues chose to use pooled data in a way that was inconsistent with how NNH (number needed to harm) should have been calculated. They used a baseline cardiovascular risk of $5.57 \%$ - not the rate of cardiovascular events in the placebo groups of their meta-analysis — as the comparison group with varenicline. Doing so created problems with their subsequent analysis, interpretation and conclusion.

The problem was in part caused by their having combined their data with other data before coming to a conclusion. They used ARE as a summary statistic, then expressed it incorrectly. This highlights why NNH, as a summary statistic itself, is inferior to ARE, as 
Woods and Caswell have implied in the previous letter. $^{2}$

On the basis of an article by Hutton, ${ }^{3}$ I believe that the interpretation of $\mathrm{NNH}$ would be better stated as the average number of comparable patients, which, if they received varenicline rather than placebo for the same period of time, would result in one patient being harmed who otherwise would not have been harmed. Since NNH is the inverse of the ARE, its correct value is $1 / 0.0024$, or 417 patients, not 28 patients, as Singh and colleagues propose. By choosing to use the latter figure as the correct NNH, they have greatly overestimated the risk produced by varenicline. The failure to use 417 from their own data as the correct $\mathrm{NNH}$ contributes to a flawed analysis, interpretation and, probably, conclusion.

In the data Singh and colleagues cite from an FDA (US Food and Drug Administration) licensure submission, ${ }^{4}$ they report that the risk with varenicline was 2.32 per 100 patient years of exposure and with placebo, 1.63 per patient years of exposure. However, the authors stop at this point and do not calculate the ARE of these data (i.e., the difference between these two incidence rates, which equals 0.69 patients per 100 patient years of exposure to varenicline, which again, like the $\mathrm{NNH}$ of 417 , is an exceedingly small number).

\section{Edward N. Squire MD MPH}

Retired allergist-immunologist, Moore Free Care Clinic, Southern Pines, NC

\section{References}

1. Singh S, Loke YK, Spangler JG, et al. Risk of serious adverse cardiovascular events associated with varenicline: a systematic review and meta-analysis. CMAJ 2011;183:1359-66.

2. Woods DJ, Caswell MD. Varenicline: quantifying the risk. CMAJ 2011;183:1404.

3. Hutton JL. Misleading statistics: the problems surrounding number needed to treat and number needed to harm. Pharm Med 2010;24:145-9.

4. Josefbert H. Approval package for: application number NDA 21-928 [clinical safety review: varenicline tartrate]. Rockville (MD): US Food and Drug Administration, Center for Drug Evaluation and Research; 2006. p. 277, 402. Available: www.accessdata.fda.gov/drugsatfda_docs/nda/2006/021928 _s000_Chantix_MedR.pdf.

CMAJ 2011. DOI:10.1503/cmaj.111-2070

The meta-analysis by Singh and colleagues tries to quantify the risk of serious adverse cardiovascular events with varenicline. ${ }^{1}$ Many representations of this risk have been disseminated, including a large increase (number needed to harm [NNH] 28) extrapolated from applying the odds ratio from an overall meta-analysis to a high-risk group and a small increase (NNH 417) extrapolated from using the absolute numbers (pooled numerators and denominators) from the overall meta-analysis.

The method of interpreting the data can give widely discrepant results. Simple pooling of absolute numbers is inaccurate because it does not account for the variations in the distribution of patients between the varenicline and placebo groups, so results are more affected by how the populations of different trials were distributed rather than the varenicline-placebo difference. However, with the formal metaanalysis, it is not clear that a single trial with a high rate of cardiovascular adverse events (about 6\% in a trial of patients with cardiovascular disease) and 13 trials with very low rates of cardiovascular events should be combined in a single meta-analysis.

To estimate the risk of serious adverse cardiovascular events in patients with stable cardiovascular disease, the best estimate may come from an analysis that is based on patients with cardiovascular disease at baseline from across these trials. Until that can be accomplished, the best estimate may be from a single trial in this population, ${ }^{2}$ in which the rates of cardiovascular adverse events were $7.04 \%$ with varenicline and $5.57 \%$ with placebo. The $\mathrm{NNH}$ would be 68 , but it was not statistically significant.

A meta-analysis of the other 13 trials found a pooled cardiovascular event rate of $0.593 \%$ with varenicline and $0.237 \%$ with placebo. The Peto odds ratio was 2.54 with a $95 \%$ confidence interval (CI) of 1.26 to 5.12. This translates into an NNH range of 103 to 1627 across the $95 \%$ CI, using a control event rate of $0.237 \%$.

The risk of serious neuropsychiatric symptoms may be of more concern, with hundreds of reported instances, including 272 completed suicides, ${ }^{3}$ but comparative evidence is limited, and no differences were found when compared with other smoking cessation medications. ${ }^{4}$

Of course, risks must be weighed against benefits, with number needed to treat (NNT) of nine for continuous smoking cessation at one year in the trial with the higher cardiovascular event rate $^{2}$ and an NNT of six to nine at 24 weeks in a Cochrane review of 10 trials. $^{5}$

\section{Brian S. Alper MD MSPH}

Editor-in-Chief, DynaMed, Medical Director, EBSCO Publishing, Ipswich, Mass.

\section{References}

1. Singh S, Loke YK, Spangler JG, et al. Risk of serious adverse cardiovascular events associated with varenicline: a systematic review and meta-analysis. CMAJ 2011;183:1359-66.

2. Rigotti NA, Pipe AL, Benowitz Nl, et al. Efficacy and safety of varenicline for smoking cessation in patients with cardiovascular disease: a randomized trial. Circulation 2010;121:221-9.

3. Institute for Safe Medication Practices. New signals for liraglutide, quetiapine and varenicline. QuarterWatch: 2010 Quarter 3. Monitoring MedWatch Reports. May 19, 2011. Available: www .ismp.org/quarterwatch/2010Q3.pdf (accessed 2011 July 18)

4. Gunnell D, Irvine D, Wise L, et al. Varenicline and suicidal behaviour: a cohort study based on data from the General Practice Research Database. BMJ 2009;339:b3805.

5. Cahill K, Stead LF, Lancaster T. Nicotine receptor partial agonists for smoking cessation. Cochrane Database Syst Rev 2011;(2):CD006103.

CMAJ 2011. DOI:10.1503/cmaj.111-2071

\section{Authors' response}

We largely agree with Blankfield ${ }^{1}$ that the known elevation of blood pressure seen with varenicline could be one explanation for the increase in serious adverse cardiovascular events. ${ }^{2}$ Other possible mechanisms include the vasoconstrictive effects of varenicline because it is a nicotinic acetylcholine receptor agonist.

Takagi and Umemoto argue about the choice of the appropriate method for pooling uncommon events. ${ }^{3}$ However, they fail to realize that RevMan automatically adds a 0.5 continuity correction to zero event studies, and this continuity correction biased their reported Mantel-Haenszel estimates toward the null, which is bordering on statistical significance (odds ratio [OR] 1.56, 95\% confidence interval 0.99-2.44). They interpret the lack of statistical significance as proof of the cardiovascular safety of varenicline. We chose the Peto-OR estimate because it is the recommended approach for uncommon events, particularly when there are trials with zero events. ${ }^{4}$ Sensitivity analyses 